\title{
Topische NSAR-Therapie Auf Wirksamkeit und Galenik kommt es an
}

\author{
Welche Chancen hat die topische \\ NSAR-Therapie? Diese Frage disku- \\ tierten Mediziner und Pharmazeu- \\ ten aus Wissenschaft und Praxis. Im \\ Fokus stand vor allem die kritische \\ Nutzen-Risiko-Bewertung der Wirk- \\ stoffe Ibuprofen und Diclofenac. Das \\ Fazit: Mit Ibuprofen als Mikrogel wird \\ die topische Schmerzbehandlung \\ präzise, schnell und verträglich.
}

Immer wieder zeigt sich: Um eine optimale Wirksamkeit zu erzielen, müssen Galenik und Wirkstoff eines Produkts auf einander abgestimmt sein. So auch beim neuen doc $^{\oplus}$ Ibuprofen Schmerzgel. Der Wirkstoff befindet sich hier in einer speziellen Mikrostruktur im unteren Nanobereich und liegt - durch die Verwendung des Hilfsstoffs Dimethylisosorbid (DMI) - vollständig gelöst vor. Dies verstärkt die Hautpenetration und ermöglicht somit die komplette Aufnahme des Wirkstoffs.

\section{Mikrogel dringt in die Haut ein}

Die gute Penetrationsfähigkeit durch die obere Hornschicht erklärte Prof. Christel Müller-Goymann von der technischen Universität Braunschweig folgendermaßen: „Alle Komponenten samt dem Arzneistoff bauen gemeinsam die Mikrogelstruktur auf. Diese Gesamtformulierung ist in der Lage,

\section{Vorsicht vor der Fehlinterpretation! \\ Altersgicht oft als Arthrose verkleidet}

\section{Gichtanfälle in jungen Jahren sind meist nicht schwer zu diagnostizie- ren. Im Alter dagegen ist der typische Gichtanfall die Ausnahme. Vor allem die Abgrenzung von der Arthrose fällt oft nicht leicht.}

Geschwollen, erwärmt und hoch schmerzhaft: Wenn das Großzehengrundgelenk plötzlich akute Beschwerden macht, ist der Gichtanfall schnell diagnostiziert - vor allem bei jungen und mittelalten Männern.

„Leider ist die Diagnose bei älteren Menschen oft etwas schwieriger", betonte Prof. Dr. Monika Reuss-Borst, Bad Kissingen. Die Probleme fangen schon damit an, dass die Gicht bei Frauen und Männern im Alter sehr viel gleichmäßiger verteilt ist: „Etwa 15-20 Jahre nach der Menopause ist die Inzidenz bei Frauen fast so hoch wie bei Männern“.

Auch die klinischen Symptome sind im Alter häufig weniger eindeutig: Die klassische Monarthritis am Bein ist dann nur noch eine von mehreren ähnlich häufigen Ausprägungsformen. „Im Alter sehen wir mehr polyartikuläre Gichterkrankungen und von Gicht betroffene Gelenke an der oberen Extremität.“

Schließlich unterscheidet sich auch der Verlauf eines Anfalls: „Die Gicht im Alter verläuft weniger akut", so Reuss-Borst. Es gebe weniger klassische Anfälle, dafür aber häufiger chronische Beschwerden.

„Das alles führt dazu, dass die Gicht im Alter immer wieder als eine scheinbare aktivierte Arthrose fehlinterpretiert wird“, so Reuss-Borst. Das ist bei akuten Beschwerden unproblematisch, weil sich beide Erkrankungen gut mit NSAR in den Griff bekommen lassen. Auf Dauer führt die Fehldiagnose freilich dazu, dass den Betroffenen eine kausale, harnsäuresenkende Therapie vorenthalten wird.

\footnotetext{
„Stoffwechselerkrankungen: Arthritis urica“, 16.09.2010, Hamburg; Veranstalter: Berlin-Chemie AG; Bericht: Philipp Grätzel von Grätz, Berlin
}

mit den obersten Schichten der Hornhaut in Wechselwirkung zu treten, so dass der Arzneistoff aus dieser Formulierung viel besser in die Haut und durch die Haut wandern kann."

Bedingt durch die rasche Freisetzung des Wirkstoffs aus der Mikrogelstruktur, tritt die schmerzhemmende Wirkung sehr schnell, nämlich innerhalb von 15 Minuten ein - deutlich schneller als bei topischem Diclofenac, das frühestens nach einer halben Stunde wirken kann. Ein weiterer positiver Effekt für die Patienten: Das Mikrogel hinterlässt keine Rückstände auf der Haut.

Expertengespräch „Chancen in der topischen NSARTherapie“, Mai 2010, Großhesselohe, Veranstalter: Hermes Arzneimittel GmbH

\section{Rosiglitazon eingestellt - wie nun behandeln?}

Vor Kurzem sprach die Europäische Arzneimittelagentur (EMA) eine Empfehlung gegen das Antidiabetikum Rosiglitazon aus. Grund dafür: der gesundheitliche Nutzen überwiegt nicht mehr die kardiovaskulären Risiken des Wirkstoffs, so die Angaben der EMA. Aufgrund dessen ordnete nun das Bundesinstitut für Arzneimittel und Medizinprodukte (BfArM) zum 01.11.2010 einen Vertriebsstopp an. Patienten, die momentan Rosiglitazon erhalten, müssen demnach auf eine andere Therapie umgestellt werden. Für Ärzte stellt sich die Frage nach einer geeigneten Alternative. Eine Möglichkeit stellt der DPP-4-Hemmer Sitagliptin (z. B. Xelevia $\left.^{\circledast}\right)$ dar. In einer Studie konnte nachgewiesen werden, dass dieser Wirkstoff in Kombination mit Metformin eine ähnliche $\mathrm{HbA}_{1 \mathrm{c}}$-Senkung erzielen konnte wie die Kombination Rosiglitazon/Metformin. Außerdem führte Sitagliptin im Gegensatz zu Rosiglitazon nicht zu einer Gewichtszunahme. 